\title{
Experimental estimation of phase noise filters efficiency for InSAR processing
}

\author{
Andrey Sosnovsky ${ }^{1, *}$ and Victor Kobernichenko ${ }^{1}$ \\ ${ }^{1}$ Ural Federal University, 620002 Mira st., 32, Ekaterinburg, Russia
}

\begin{abstract}
The experimental study of the efficiency of interferometric data processing algorithms for synthetic aperture space radars is complicated by the fact that a quantitative result can be obtained only after the completion of the entire technological chain. The efficiency of the first stages of interferometric processing - incoherent accumulation and suppression of phase noise-has been studied on the basis of the method of inverse transformation of standards previously proposed by the authors. The results of estimation of accuracy of digital elevation model construction for ALOS PALSAR data with different base lengths and polarizations of the probing signal are presented.
\end{abstract}

\section{Introduction}

A widespread application of interferometric data processing (InSAR and DInSAR technologies) for obtaining Earth surface elevation and its changes has become one of the main trends in the development of modern radar systems of space observation, along with the implementation of ultra-high spatial resolution modes and shooting in full polarization basis [1-3]. The essence of the method of space radar interferometry is the joint processing of phase fields obtained by imaging the same area simultaneously by radar with two systems (cross-track interferometry), or with one antenna and two orbit passes (repeat-track interferometry) to extract information about the shape of the Earth's surface relief and/or its changes. It combines the high accuracy of the phase measurements with the high resolution of synthetic aperture radars (SAR).

The InSAR processing chain for digital elevation model (DEM) obtaining includes the following steps: a formation of a pair of complex radar images, spatial combination of radar images of the same surface area; generation of an interferogram by element-by-element complex multiplication of two radars; compensation of the phase trend from the reference surface (interferogram flattening); incoherent accumulation (multilooking); estimation of data coherence; phase noise suppression; elimination of phase ambiguity (phase unwrapping); conversion of unwrapped (absolute) phases into the the elevations (or relief displacements), and geocoding (data transformation from the SAR image coordinate system to any map projection).

Complexity of the implementation and interpretation of the results for three main stages of interferometric processing, i.e. multilooking, phase noise suppression and phase unwrapping, makes it difficult to use InSAR data to solve the needs of geodesy and

\footnotetext{
${ }^{*}$ Corresponding author: $\underline{\text { sav83@e1.ru }}$
} 
cartography. Radar image mulitlooking represents the summation of its neighboring samples with equal weights and allows to reduce the level of interferometric phase fluctuations with simultaneous degradation of spatial resolution. Also it allows one to adjust the azimuthal and slant range spatial resolutions of the interferogram and accelerate the further steps of the interferometric processing. Phase noise suppression is a critical step in interferometric processing, because regardless of the imaging conditions, the initial phase noise level makes it impossible to further restore the absolute phase. Suppression is realized by two-dimensional linear or adaptive filters.

This work is devoted to development of an estimation technique for the efficiency evaluation of phase noise suppression algorithms on the InSAR interferograms using various filters. Experimental studies were conducted on the example of data obtained by the ALOS PALSAR radar.

\section{Estimation technique for the efficiency evaluation of the phase noise suppression algorithms}

The phase difference of signals obtained by radar interferometric survey has 4 components [1-3]:

$$
\Delta \varphi_{m, n}=W\left\{\frac{4 \pi}{\lambda} \Delta r_{m, n}+\frac{4 \pi}{\lambda} D_{m, n}+\Delta \psi_{\text {atm } m, n}+\Delta \varphi_{\text {noise } m, n}\right\},
$$

where $\Delta r_{m, n}$ is the difference of distances from the surface element to the phase centers of the synthesized apertures during the first and second passes, $\Delta D_{m, n}$ is the displacement of the surface element during the time between passes, $\Delta \psi_{\text {atm } m, n}$ is the phase difference caused by the signal propagation in the atmosphere, $\Delta \varphi_{\text {noise } m, n}$ is the phase noise, $m, n$ are the discrete coordinates of the interferometric image (interferogram) elements, $W\{\}$ is the phase wrapping operator. The first term in (1) describes the "topographic phase", which deals with the height of the surface element. The direct calculation of the interferometric difference is as follows:

$$
\Delta \varphi_{m, n}=\arg \left\{\dot{Z}_{1 m, n} \cdot \bar{Z}_{2 m, n}\right\},
$$

where $\dot{Z}_{1 m, n}, \dot{Z}_{2 m, n}$ are the spatially combined first (master) and second (slave) complex radar images.

Phase noise on interferograms $\Delta \varphi_{\text {noise }}$ appears due to the variability of the electrical properties of the surface, atmospheric and electronic equipment noises. In the absence of incoherent accumulation (multilooking) of image samples, its distribution density is described by the following expression

$$
p(\Delta \varphi)=\frac{1-\gamma^{2}}{2 \pi} \frac{1}{1-\gamma^{2} \cos ^{2}\left(\Delta \varphi-\Delta \varphi_{0}\right)}\left(\frac{\gamma \cos \left(\Delta \varphi-\Delta \varphi_{0}\right) \arccos \left(-\gamma \cos \left(\Delta \varphi-\Delta \varphi_{0}\right)\right)}{\sqrt{1-\gamma^{2} \cos ^{2}\left(\Delta \varphi-\Delta \varphi_{0}\right)}}+1\right)
$$

where $\Delta \varphi_{0}$ is the mathematical expectation of the interferometric phase, which is approximately corresponds to the topographic phase of the terrain. The effect of multilooking on the phase distribution density is discussed in detail in [1,3] and it is expressed in a decrease in the phase variance proportionally to the number of looks.

Phase noise suppression results in a smoothed interferogram $\Delta \varphi_{m, n}^{f}$ :

$$
\Delta \varphi_{m, n}^{f}=\arg \left\{\dot{\mathbb{F}}_{m, n}\left[e^{j \Delta \varphi_{m, n}}\right]\right\}
$$


where $\dot{\mathbb{F}}_{m, n}[]$ is the filter operator (including non-stationary and nonlinear cases). The Goldstein adaptive frequency-domain filter [5] is the most widely used among filtration methods. It calculates the two-dimensional FFT spectrum of the interferogram block, and weights it as follows

$$
\dot{G}_{f}(k, l)=|\dot{G}(k, l)|^{\alpha[1-\overline{\gamma(k, l)}]} \cdot \dot{G}(k, l),
$$

where $\dot{G}(k, l)$ is the two-dimensional spectrum of the processed interferogram block, from which the block of smoothed interferogram is restored by the inverse two-dimensional FFT, $\overline{\gamma(k, l)}$ is the average coherence of the scene within the processed block, $\alpha$ is the coefficient that enhances the effect of the coherence value on the filter "strength". The filter operates in overlapping blocks, after processing adjacent blocks are stitched using a weighing function.

As a rule, it is possible to evaluate the efficiency of phase noise suppression for real scenes (in terms of the precision of the interferometric processing result) after the completion of the entire interferometric processing chain ("end-to-end evaluation") by comparison of the result (the elevation matrix or its individual elements) with the reference elevation data obtained by ground measurements. Regular (GRID) and irregular (TIN) digital elevation models, arrays of of individual terrain points coordinates (elevation points of digital topographic maps) can act as reference elevation data. From the points of view of ease of imaged and reference data fitting and the adequacy of resulting estimates the reference data represented as an array of of elevation points $\left\{H_{i}\right\}$ of digital topographic maps are best suited for the implementation of the proposed estimation technique. In contrast to the matrix reference DEM, the use of arrays of individual points eliminates the impact of the scene areas that are not provided with reference information (i.e. water surfaces and areas of open pit mining) on the precision estimation results.

However, the precision estimates obtained for the whole interferometric processing chain are determined not only by the efficiency of phase noise suppression, but also by the efficiency of phase unwrapping, and by geocoding errors. That makes it difficult to compare the effectiveness of different phase noise suppression methods and algorithm parameters on the final result. To resolve this difficulty, the authors previously [6] proposed a method of reverse reference data transformation to obtain an absolute phase reference. The estimation technique on the method includes the following steps:

- transformation of the reference heights coordinates $\left\{B_{i}, L_{i}, H_{i}\right\}$ from geocentric coordinate system into a radar image coordinate system $\left\{m_{i}, n_{i}, H_{i}\right\}$ using the rangedoppler equations (inverse geocoding) [4];

- horizontal alignment of the converted reference height points with the amplitude radar image to compensate the orbital errors;

- vertical alignment of the converted reference height points and the restored absolute phase.

Horizontal alignment is implemented by means radar benchmarks or other reference elements, which are confidently identifiable at the radar image. The vertical alignment is implemented using an unwrapped phase, which is obtained once with the utilization of the phase noise banpass filter (for example, Gaussian filter with a bandwidth, which is approximately equal to the width of the interferogram spectrum) or an adaptive filter and any phase unwrapping algorithm. Under ideal conditions, the restored absolute phase $\Delta \psi_{i}$ must be proportional to the estimated surface height $\hat{H}_{i}$ :

$$
\hat{H}_{i}=\frac{h_{A i}}{2 \pi} \hat{\Psi}_{i},
$$


however, due to inaccuracies in determining the orbital parameters and, as a consequence, inaccuracies in the calculation of ambiguity heights $h_{A i}$, the restored absolute phase will have additional slope angles (skews) and, possibly, additional scale distortion relative to the reference surface. Thus, to correctly estimate the accuracy of the restored absolute phase, it is necessary to convert the reference absolute phases in the way, that the ambiguity height calculation error and the skews were compensated. To do this, the reference heights $\Psi_{i}^{0}$ must be converted to reference absolute phases, taking into account the correction factors:

$$
\Psi_{i}^{0}=\hat{k}_{H} H_{i}+\hat{k}_{m} m_{i}+\hat{k}_{n} n_{i},
$$

where $\hat{k}_{H}, \hat{k}_{m}$ and $\hat{k}_{n}$ are correction factors for height scale $\left(\hat{k}_{H} \sim 2 \pi / h_{A}\right)$ and phase skew angles along the coordinates $m$ and $n$. To calculate the factors, the redefined system of equations in the following form is used:

$$
\left\{\begin{array}{l}
\hat{\Psi}_{1}=\hat{k}_{H} H_{1}+\hat{k}_{m} m_{1}+\hat{k}_{n} n_{1} \\
\cdots \\
\hat{\Psi}_{N}=\hat{k}_{H} H_{N}+\hat{k}_{m} m_{N}+\hat{k}_{n} n_{N}
\end{array},\right.
$$

which is further solved by the least squares method with respect to $\hat{k}_{H}, \hat{k}_{m}$ and $\hat{k}_{n}$ ( $N$ is the number of reference points).

An example of the result of vertical alignment is shown in Fig. 1, from which it can be seen that before the vertival alingment the difference between the restored and the reference phases has a significant skew (Fig. 1a). After alignment, the skews turn to be suppressed, and standard deviation of the difference between the reference and restored absolute phases $\delta \psi$ decreases one and a half times (Fig. 2).

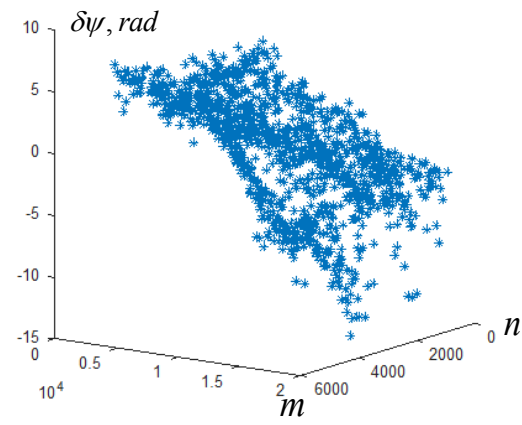

$a$

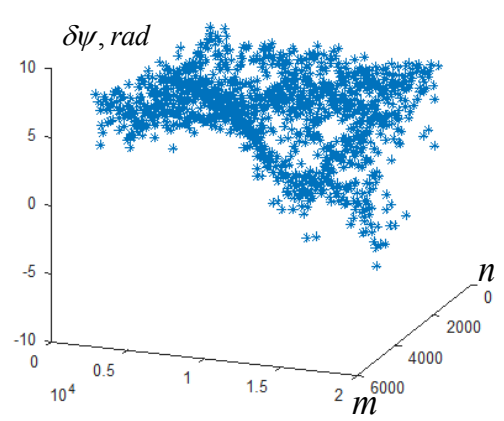

$b$

Fig. 1. The spatial distribution of the recovered absolute phase and the absolute reference phase differences: a) before vertical alingment; b) after vertical alingment.
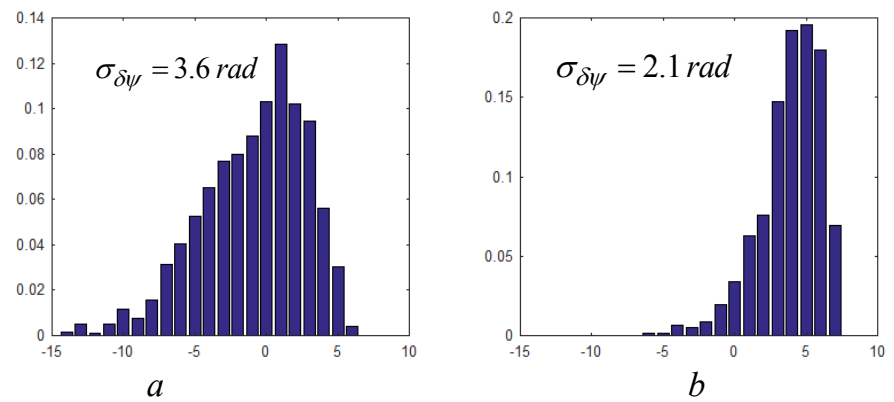

Fig. 2. Histograms of the recovered absolute phase and the absolute reference phase differences: a) before vertical alingment; b) after vertical alingment. 
To assess the quality of phase noise suppression, the standard deviation criterion of the difference phase angle $\delta \varphi$ between the smoothed and reference phases $\sigma_{\delta \varphi}$ is used, which is calculated in the following way:

$$
\begin{aligned}
& \sigma_{\delta \varphi}=\sqrt{\frac{\sum_{i}\left(\delta \varphi_{i}\right)^{2}}{N-1},} \\
& \delta \varphi_{i}=\arg \left[\frac{e^{j \Delta \varphi_{i}} \cdot e^{-j \Psi_{i}^{0}}}{e^{j \overline{\Delta \varphi_{i}}} \cdot e^{-j \Psi_{i}^{0}}}\right],
\end{aligned}
$$

Further evaluation of the phase noise suppression algorithms efficiency includes the following steps:

1. Experimental processing of interferograms. At this stage, a series of calculations for the stages of multilooking with coefficients $N_{A j} \times N_{R j}$ and phase noise suppression by the studied algorithms and their parameters $p_{i j}$ (where $i$ is the number of the algorithm, and $j$ is the value of the parameter) are sequentially carried out with fixation of the $\sigma_{\delta \varphi i, j}$ values. It should be noted that a separate study of the multilooking effectiveness is impractical, since the high level of remaining phase noise does not make it possible to obtain meaningful results.

2. Plotting the experimental characteristics. The results of $\sigma_{\delta \varphi i, j}$ measurements are grouped into families of graphs $\left.\sigma_{\delta \varphi}\left(p_{i j}\right)\right|_{N_{A j} \times N_{R j}}$, i.e. the representation of the $\sigma_{\delta \varphi}$ dependence on the $j$-th parameter value with the fixed multilooking coefficient value.

3. Analysis of the obtained characteristics. The analysis is carried out in order to determine the algorithms that provide the minimum value of the differential phase angle deviation $\sigma_{\delta \varphi}$ and appropriate algorithms and their parameters values.

\section{Experimental results}

The experimental studies used the ALOS PALSAR radar data, which are represented by five scenes (interferometric radar pairs) of three test sites. Scenes No. 1 and No. 2 were obtained in two polarizations (HH and HV-polarization, respectively), the phase has an ambiguity height of $h_{A \max }=18 \mathrm{~m}$ (in the middle side); scenes No. 3-5 were obtained in single polarization ( $\mathrm{HH}$, so it has 2 times better range resolution), ambiguity heights are $125 \mathrm{~m}, 42 \mathrm{~m}$ and $62 \mathrm{~m}$, respectively (table 1).

The reference data set includes more than 2.5 thousand height points with vertical accuracy not worse than $0.5 \mathrm{~m}$.

Table 1. Experimental data characteristics.

\begin{tabular}{|c|l|c|c|c|c|c|}
\hline $\begin{array}{c}\text { Scen } \\
\mathbf{e} \\
\text { num } \\
\text {-ber }\end{array}$ & \multicolumn{1}{|c|}{ Surface type } & $\begin{array}{c}\text { Imaging } \\
\mathbf{m o d e}\end{array}$ & $\begin{array}{c}h_{A \text { max }}, \\
\mathbf{m}\end{array}$ & $\begin{array}{c}\text { Mean scene } \\
\text { coherence } \\
\bar{\gamma}\end{array}$ & $\begin{array}{c}\text { Normal / critical } \\
\text { baseline } \\
B_{\perp} / B_{\perp c r}, \mathbf{k m}\end{array}$ & $\begin{array}{c}\text { Interferogram } \\
\text { spectral bandwidth } \\
\Delta f_{a z} \times \Delta f_{r g}, \mathbf{p i x} .\end{array}$ \\
\hline 1 & $\begin{array}{l}\text { Hilly relief, forest and } \\
\text { grassy vegetation }\end{array}$ & FBD-HH & 18 & 0,28 & $3,4 / 6,6$ & $160 \times 133$ \\
\hline 2 & $---------/ /-------$ & FBD-HV & 18 & 0,21 & $3,4 / 6,6$ & $197 \times 183$ \\
\hline 3 & ---------//------ & FBS & 125 & 0,38 & $0,51 / 13,1$ & $48 \times 33$ \\
\hline 4 & $\begin{array}{l}\text { Hilly relief, forest and } \\
\text { grassy vegetation, urban } \\
\text { development }\end{array}$ & FBS & 42 & 0,26 & $2,0 / 19,2$ & $112 \times 117$ \\
\hline 5 & $\begin{array}{l}\text { Low mountainous relief, } \\
\text { forest vegetation }\end{array}$ & FBS & 62 & 0,19 & $1,4 / 19,2$ & $191 \times 178$ \\
\hline
\end{tabular}


The efficiency of the following phase noise suppression algorithms was investigated:

1) averaging in the local window (the studied parameter is the size of the moving square averaging window).

2) frequency filter with Gaussian frequency response (the studied parameter is the cutoff frequency).

3) Goldstein spectral adaptive filter (the studied parameters are the block size and adaptation coefficient $\alpha$ value).

The dependences of the $\sigma_{\delta \varphi}$ are selectively presented in figures 3-5. The overall results are presented in table 2.

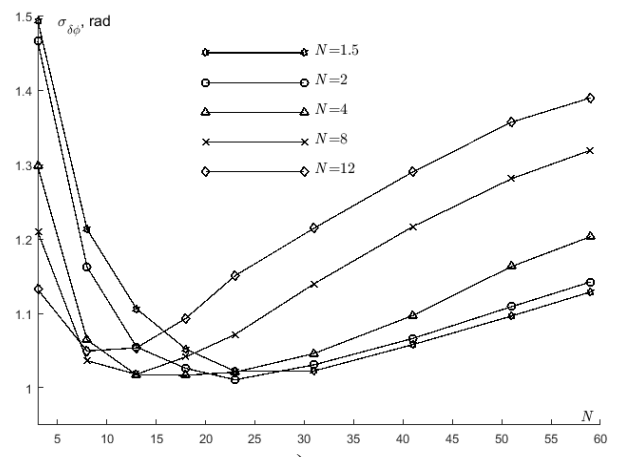

a)

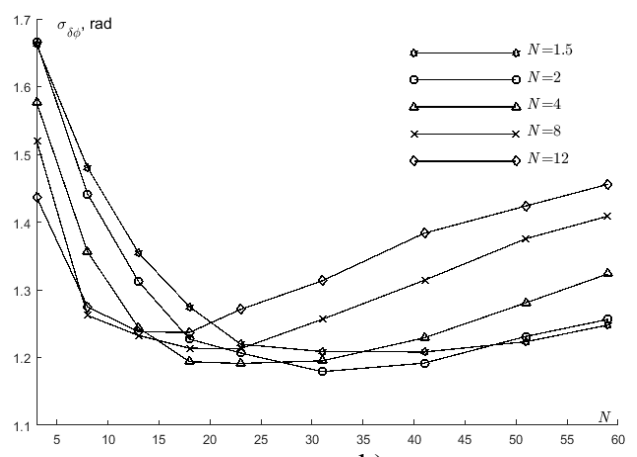

b)

Fig. 3. $\sigma_{\delta \varphi}$ dependencies on the window size for the averaging algorithm: a) scene N 1; b) scene N 2 .

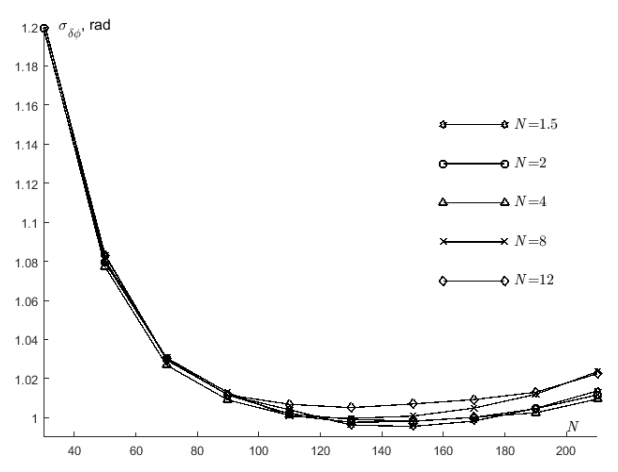

a)

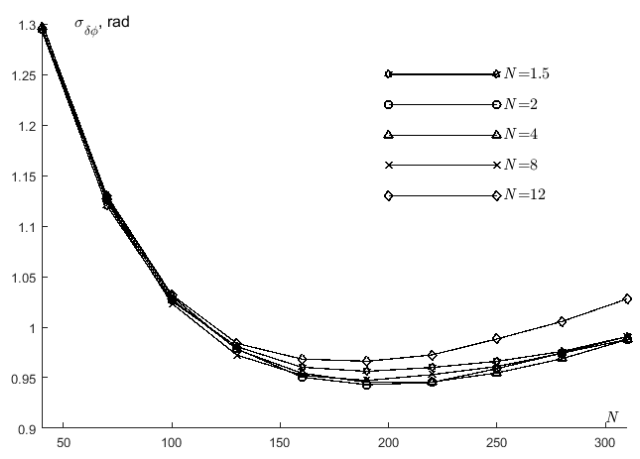

b)

Fig. 4. $\sigma_{\delta \varphi}$ dependencies on the cutoff frequency (pix) for spectral Gaussian filter: a) scene $\mathrm{N} 1$; b) scene N 5.

Table 2. Optimal filteration algorithms parameters.

\begin{tabular}{|c|c|c|c|c|c|c|c|c|c|}
\hline \multirow{2}{*}{$\begin{array}{c}\text { Scene } \\
\text { num- } \\
\text { ber }\end{array}$} & $\sigma_{\delta \varphi \min }$ & $\begin{array}{c}\text { Averaging filter } \\
\text { window } \\
\text { size, } \\
\text { pix. }\end{array}$ & $\begin{array}{c}\text { Optimal } \\
\text { azimuthal } \\
\text { multi- } \\
\text { looking } \\
\text { coef. }\end{array}$ & $\sigma_{\delta \varphi \min }$ & $\begin{array}{c}\text { Optimal } \\
\text { band- } \\
\text { width, } \\
\text { pix. }\end{array}$ & $\begin{array}{c}\text { Optimal } \\
\text { azimuthal } \\
\text { multi- } \\
\text { looking } \\
\text { coef. }\end{array}$ & $\sigma_{\delta \varphi \text { min }}$ & $\begin{array}{c}\text { Gaussian filter } \\
\text { mal } \\
\text { block } \\
\text { size, } \\
\text { pix. }\end{array}$ & $\begin{array}{c}\text { Gpti- } \\
\text { mal } \\
\alpha \\
\text { value }\end{array}$ \\
\hline 1 & 1.01 & $23 \times 23$ & 2 & 1.00 & 150 & 1.5 & 1.01 & 45 & 4.0 \\
\hline 2 & 1.18 & $31 \times 31$ & 2 & 1.17 & 90 & 2 & 1.18 & 50 & 4.0 \\
\hline 3 & 0.45 & $61 \times 61$ & 2 & 0.45 & 50 & 2 & 0.45 & 75 & 4.0 \\
\hline 4 & 0.94 & $13 \times 13$ & 4 & 0.93 & 190 & 1.5 & 0.95 & 35 & 4.0 \\
\hline 5 & 1.00 & $13 \times 13$ & 4 & 0.97 & 190 & 2 & 1.00 & 35 & 4.0 \\
\hline
\end{tabular}




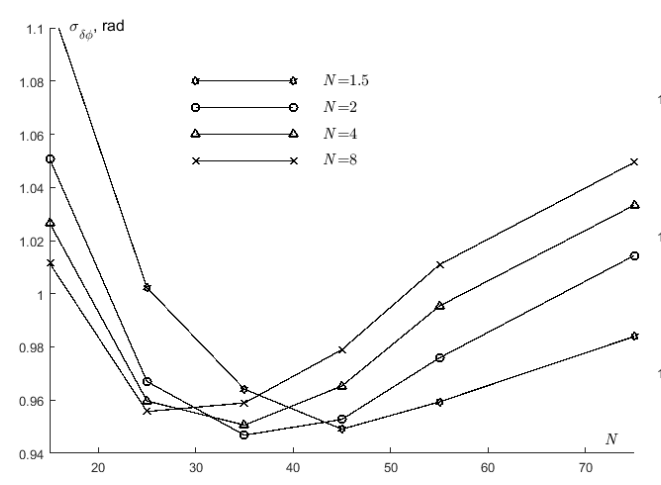

a)

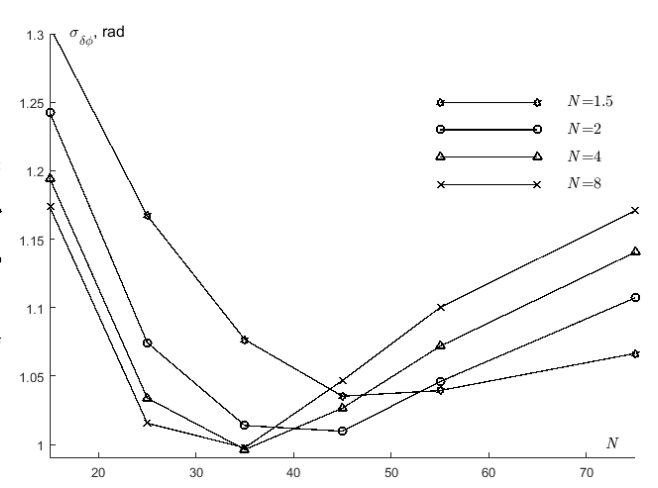

b)

Fig. 5. $\sigma_{\delta \varphi}$ dependencies on the filter block size for different $\alpha$ values for the Goldstein algorithm: a) scene $\mathrm{N} 4$; b) scene $\mathrm{N} 5$.

Obtained minimal $\sigma_{\delta \varphi}$ values ranged from 0.45 to $1.17 \mathrm{rad}$ for different scenes, which corresponds to heights standard deviation from 2.9 to $9.1 \mathrm{~m}$. The differences for the different algorithms are hundredths of a radian, which indicates their approximate equivalence. However, for the averaging and Gaussian filters, the optimal mode is achieved in a relatively narrow range of variable parameters, and the optimal values of the parameters themselves depend significantly on the properties of a particular processed scene. For the Goldstein filter, the minimum is usually achieved in the block size range of 35-45 for the majority of scenes being processed (excepting the scene with the extremely short baseline).

\section{Conclusion}

The tecnnique for experimental estimation of the phase noise suppression efficiency in interferometric processing using different algorithms and their parameters is developed. The efficiency of phase noise suppression for ALOS PALSAR radar data is investigated. It is shown that for various scenes obtained for hilly and low-mountainous terrain, the standard deviation of the interferometric phase from the reference values is $0.45 . .1 .20 \mathrm{rad}$., which corresponds to the height standard deviation from 2.9 to $9.1 \mathrm{~m}$. Different phase noise filters can achieve approximately the same minimum error values, but non-adaptive filters have optimal parameter values determined by the properties of the scene, while the adaptive filter keeps the optimal parameter values approximately the same for different scenes.

The work was supported by Act 211 Government of the Russian Federation, contract № 02.A03.21.0006. The authors thank JSC "Uralgeoinform" (Ekaterinburg) and Sovzond Company for radar imagery delivered.

\section{References}

1. R. Bamler, P. Hartl, Synthetic aperture radar interferometry, Inverse Problems, 14(4), pp. r1-r54. (1998)

2. I.R. Joughin, F.K. Li, S.N. Madsen, E. Rodrigues, Synthetic Aperture Radar Interferometry, Proceedings of the IEEE, 88(3), pp. 333-382 (2000)

3. R.F. Hanssen. Radar interferometry. Data interpretation and error analysis (Kluwer academic publishers, 2001) 
4. D. Small, C. Werner, D. Nuesch, Geocoding and validation of ERS-1 InSAR-derived digital elevation models, EARSeL Advances in Remote Sensing, 4(2), pp. 26-39 (1995)

5. I. Baran, M.P. Stewart, B.M. Kampes, Z. Perski, A Modification to the Goldstein Radar Interferogram Filter. IEEE Transactions on Geoscience and Remote Sensing, 41(9), pp. 2114-2118 (2003)

6. A.V. Sosnovsky, V.G. Kobernichenko, A technique for evaluation of InSAR processing stages efficiency. Proc. of CriMiCo'2016, Sevastopol, 1, pp. 2716-2722 (2016) 